FiLdes, P. (1956). J. gen. Microbiol. 15, 636-642

\title{
Production of Tryptophan by Salmonella typhi and Escherichia coli
}

\author{
BY P. FILDES \\ The Virus Unit of the British Empire Cancer Campaign, \\ Sir William Dunn School of Pathology, Oxford
}

SUMMARY : In order to determine whether Salmonella typhi in growth produces free tryptophan in excess, extracts and cultures were tested by various microbiological methods. A positive result was seldom seen, but when excess indole was added to the culture, comparatively large yields of tryptophan were obtained with all strains examined except one in which there was some disability in the indole $\rightarrow$ tryptophan process. Escherichia coli behaved in the same way, the free tryptophan produced being preserved owing to inhibition of tryptophanase by the indole added.

Logie (1919-20) was the first to show that certain bacteria could synthesize tryptophan. They were grown on a medium in which $\mathbf{N H}_{3}$ was the only source of nitrogen and when tested by the glyoxylic method gave a positive reaction for tryptophan. Fildes, Gladstone \& Knight (1933) confirmed this result. An extensive literature has since developed on the details of the biosynthesis of tryptophan and its relationship to bacterial nutrition. This need not be discussed. So far as the biosynthetic chain is concerned, it has been proved by chemical and biological methods that anthranilic acid, indole and serine are concerned in the process, but the final product tryptophan has not been determined satisfactorily until recently, except in the case of the mould Neurospora crassa. Tatum \& Bonner (1944) made short reference to production of tryptophan according to colour tests from indole and serine by Escherichia coli suspensions, but Fildes (1945) failed with Salmonella typhi and, though Fildes \& Rydon (1947) found that 4-methyltryptophan inhibited the further use of tryptophan and so might be expected to cause accumulation of tryptophan in the culture, this did not occur according to colour tests.

During a study of a bacteriophage which required tryptophan for absorption, it became necessary to settle whether free tryptophan was, in fact, present in or on the typhoid bacteria in use. Since, previously, chemical methods of detection had failed, microbiological methods were now used.

Two series of experiments were carried out. In the first the bacteria were extracted with buffer at $100^{\circ}$ and the extracts assayed for tryptophan by a microbiological growth method. In the second series use was made of the Escherichia coli mutants now available which have an essential requirement for tryptophan in growth. These were used in two ways:

(a) Along the lines of Winkler, van Doorn \& Royers (1952) to show that these coli mutants can grow syntrophically with Salmonella typhi, although without the symbiosis they can only grow with added tryptophan. The fact 
of the growth of the coli mutant syntrophically with another bacterium is taken to imply that the other bacterium has produced free tryptophan.

(b) To produce a 'satellite' effect, i.e. to determine whether a patch of the bacterium under test for production of tryptophan will produce satellite colonies or zones on the surrounding lawn of mutant coli organisms.

\section{METHODS}

Organisms used. Salmonella typhi: 'Bact 1', a strain without synthetic block; O901 R, a rough mutant of the well-known strain O901, which has a synthetic block immediately below indole; 1808, described later; others as stated later. Escherichia coli: strain B, the American phage-test strain; 7/4 a mutant blocked above indole, supplied by Professor D. D. Woods; 366, a similar mutant of $\boldsymbol{E}$. coli strain B, supplied by Professor K. C. Winkler.

Media. 'Defined' medium refers to a simple ammonia+glucose + salts medium containing $\mathrm{M} / \mathbf{1 0 , 0 0 0}$ cystine.

'Casein medium' contained buffer, 'vitamin-free' hydrolysed casein and glucose.

'Lactose-neutral red' medium was a nutrient agar based on the wellknown MacConkey medium but without bile salts.

\section{EXPERIMENTAL}

Extraction of tryptophan from Salmonella typhi

Experiments were made to find whether tryptophan could be extracted from the bacteria merely by heating with water or neutral buffer at $100^{\circ}$. Gale (1947) found that other amino acids could thus be extracted from Gram-positive bacteria.

Exp. 1. Extraction of tryptophan from Salmonella typhi by buffer at $100^{\ulcorner}$. One litre defined medium was inoculated with $S$. typhi Bact. 1 and agitated overnight at $37^{\circ}$. Next morning the opacity (Spekker) reading was $1 \cdot 300$, roughly equivalent to $2 \mathrm{mg}$. dry wt. bacteria $/ \mathrm{ml}$; $880 \mathrm{ml}$. were centrifuged and the deposit made up in $\mathrm{M} / 30$ buffer ( $\mathrm{pH} \mathrm{7 \cdot 2}$ ) to $\mathbf{1 0} \mathrm{ml}$. Thus the concentration of bacteria was now equivalent to $c .0 \cdot 2 \mathrm{~g}$. dry wt./ml. This suspension was then heated to $100^{\circ}$ for $30 \mathrm{~min}$., centrifuged and the supernatant fluid retained for estimation of the tryptophan content by the usual microbiological growth method. Seven different extracts were made as indicated in Table 1, differing in the concentration of bacteria in the extracts and in extraction time. Six were derived from $S$. typhi Bact. 1 and one from strain $0901 \mathrm{R}$. This latter organism was grown with tryptophan and was washed four times before extraction.

The organism used as growth-test object in the assay was Salmonella typhi O901 R. This was grown overnight in defined medium + tryptophan. It was then washed 4 times in defined medium without tryptophan. The use of strain $0901 \mathrm{R}$ as a test for tryptophan is, of course, open to criticism because it grows also in the presence of indole. It was, however, used as a convenience. 
The criticism was met by showing that steam-distilled extracts did not differ significantly from undistilled and therefore did not contain indole. Further, one of the extracts was of O901 R itself, which does not synthesize indole. In the assay, growth due to added extracts or tryptophan was estimated by opacity (Spekker) and converted to $\mu \mathrm{g}$. tryptophan $/ \mathrm{ml}$. from a standard curve.

Table 1 shows that the extracts contained a material with the biological action of tryptophan. This was present both in bacteria without synthetic blocks and in $0901 \mathrm{R}$ which is blocked below indole.

Table 1. Estimation of tryptophan in extracts of Salmonella typhi Bact. 1 (1 to 6) and of S. typhi strain O901 R (7)

\begin{tabular}{|c|c|c|c|}
\hline $\begin{array}{c}\text { Extract } \\
\text { no. }\end{array}$ & $\begin{array}{c}\text { Concentration } \\
\text { of extract }\end{array}$ & $\begin{array}{c}\text { Extraction } \\
\text { time } \\
\text { (min.) }\end{array}$ & $\begin{array}{c}\text { L-tryptophan } \\
\text { in undiluted extract } \\
(\mu \mathrm{g} . / \mathrm{ml} .)\end{array}$ \\
\hline 1 & $10 / 1$ & 5 & $0 \cdot 33 \pm 33 \%$ \\
\hline 2 & $88 / 1$ & 7 & Doubtful \\
\hline 3 & $88 / 1$ & 7 & $0.58 \pm 24 \%$ \\
\hline 4 & $88 / 1$ & 7 & Doubtful \\
\hline $\mathbf{5}$ & $88 / 1$ & 30 & $1 \cdot 49 \pm 8 \%$ \\
\hline 6 & $66 / 1$ & 30 & $1 \cdot 26 \pm 9 \%$ \\
\hline$\left(\begin{array}{c}7 \\
(0901 R)\end{array}\right.$ & $88 / 1$ & 30 & $1 \cdot 28 \pm 9 \%$ \\
\hline
\end{tabular}

Syntrophism of tryptophan-requiring Escherichia coli with Salmonella typhi

The purpose of these experiments was to prove the presence of free tryptophan, not only in or on Salmonella typhi, but diffusing from it. The free tryptophan was to be detected by mixing with the culture a tryptophanrequiring coli mutant as indicator. These mutants have a synthetic block above indole and therefore accumulate indole in the culture. If syntrophic growth took place between the coli mutant and a typhoid strain under test in a medium free from indole or tryptophan, it should be an indication that the tryptophan required by the coli mutant was derived from the typhoid organisms.

The evidence of successful syntrophic growth was therefore as follows:

(1) The mixed culture in an indole- and tryptophan-free medium should accumulate indole and continue to do so on serial passage.

(2) The culture when plated on nutrient agar containing lactose and neutral red should grow differentiated colonies of coli and typhoid bacilli which could be isolated and tested to show that the bacteria were in the same metabolic condition as initially.

The following is an example of this type of experiment:

Exp. 2. Syntrophism between Escherichia coli $7 / 4$ and Salmonella typhi O901 R. S. typhi $0901 \mathrm{R}$ and $E$. coli $7 / 4$ were grown overnight in casein medium $+M / 10,000$ indole or tryptophan, respectively. Next day, 1 drop of each culture was transferred separately to casein medium alone and for the syntrophic growth 1 drop of each together to casein medium. In $24 \mathrm{hr}$. the combined tube only had grown and one loop was taken to casein medium (1st transfer). Next day this had grown. The supernatant fluid contained indole 
(Ehrlich's reagent) and both coli and typhoid organisms grew on the lactose + neutral red plates. These transfers were repeated 4 times and the last plating showed both typhoid and coli organisms. Colonies of the coli from the final neutral red plate were diluted and one loop taken to casein medium alone, to casein + indole and to casein + tryptophan. Growth appeared in all, showing that back-mutation had taken place. In the tube containing casein alone there was an accumulation of indole. This could only have been brought about by a coli back-mutant supplying the tryptophan.

This being so it might be argued that the growth of Escherichia coli $7 / 4$ in syntrophism with the typhoid organism was due to tryptophan derived from a coli back-mutant and not from the typhoid bacilli. The experiment was therefore inconclusive so far as the typhoid organism was concerned.

The experiment was repeated using however Salmonella typhi Bact. 1 in place of $S$. typhi $0901 \mathrm{R}$. The same procedure was followed with the same result to the 4 th transfer. The coli and typhoid organisms were then isolated and each lightly inoculated into casein medium alone, casein + indole and casein +tryptophan. The coli grew only in tryptophan (indole + ) while $S$. typhi Bact. 1, having no block, grew in all the media as usual. In this case therefore it seemed clear that tryptophan derived from the $S$. typhi Bact 1 was the cause of the growth of Escherichia coli 7/4. This type of experiment is, however, apt to be abortive because of chance growth of coli back-mutants (cf. Winkler, van Doorn \& Royers, 1952).

\section{Satellitism experiments}

In these experiments tryptophan produced by an organism was to be detected on an agar plate by production of a zone of growth of a tryptophanrequiring coli lawn surrounding an implanted patch of the organism under test. The Escherichia coli mutant 7/4 was used as the lawn and 9 different strains of Salmonella typhi: Vi strains, A, T, 1808; Smooth non-Vi, O901 S, P; Rough, 0901 R, Bact. 1, ViIR. Bact. 1 had no synthetic block, but the others (except 1808) were blocked immediately below indole. Strain 1808 is discussed later.

All strains except 1808 produced zones of growth and therefore were judged to produce tryptophan. The procedure was as follows: Escherichia coli 7/4 was grown overnight in defined medium + tryptophan $(\mathrm{M} / 10,000)$, washed twice in defined medium, diluted in the same to about $10^{7}$ organisms $/ \mathrm{ml}$. and $0.5 \mathrm{ml}$. of this dilution flooded on to a casein plate. The fluid was then drained off and the plate dried on a hot-plate. On this lawn were dropped $(c .1 / 40 \mathrm{ml}$.) the typhoid cultures, each on a separate plate. They had been grown in casein medium with or without indole as necessary and washed once without indole. After drying the drops, the plates were incubated. Next day, the lawn remained free from obvious growth (or with a few back-mutants) but the plates had an odour of indole. The drops of suspension on the lawn had grown, those blocked using indole produced by the lawn. Controls dropped on a plate without lawn grew only in the case of $S$. typhi Bact. 1. In 2 days or perhaps later, a hazy zone appeared on the periphery of the patches which increased slowly to a well-marked halo. The controls without lawn showed nothing 
similar. On examination a halo was found to consist of an increased surface growth and a subculture contained exclusively $E$. coli $7 / 4$. Pl. 1, figs. 1 and 2, show the effect with $S$. typhi $0901 \mathrm{R}$; figs. 3 and 4 with $S$. typhi Bact. 1.

These experiments were in agreement with a conclusion that Salmonella typhi produced excess tryptophan in the course of growth, even though the amount produced, as judged by the effect, was surprising in view of previous inability to obtain positive results. The minimal amount could be estimated roughly by measuring the diameters of the haloes and comparing with the rings produced by known quantities of tryptophan using the usual punched-out pit method. On this basis each drop of culture produced some $0 \cdot 5-5 \cdot 0 \mu \mathrm{g}$. L-tryptophan. In fact, of course, with dilute tryptophan solutions in the pits the haloes were mere films, whereas with drops of culture there was a continuous production which resulted in a thick film without, however, increase in diameter. Thus the amount of tryptophan produced during growth was probably higher than this assessment suggested.

\section{The effect of indole}

Salmonella typhi strain 1808 was frequently isolated in 1946 in an epidemic in Wales. It has a nutritional disability which has not been fully analysed. In the absence of indole or tryptophan there is no growth, but with indole growth takes place slowly, though much more rapidly with tryptophan. In spite of the indole produced by lawns of Escherichia coli $7 / 4, S$.typhi strain 1808 dropped on grew very little and produced no halo. It seemed that there was some difficulty in the synthesis of tryptophan and that this might be great enough to allow strain 1808 to be used as a lawn for indicating tryptophan-production. It had been noted that back-mutants of $E$. coli $7 / 4$ had shown marked haloes on a lawn of $E$. coli $7 / 4$ itself ( $\mathrm{Pl} .1$, fig. 5) and it was concluded that $E$. coli B also must excrete tryptophan. E. coli B was therefore tried to test the value as a lawn of $S$. typhi 1808; but no haloes appeared in 6 days. From this it could be argued that a lawn of $S$. typhi 1808 was not stimulated by tryptophan from $E$. coli B, or that $E$. coli B did not excrete tryptophan. It was, however, recognized that the conditions of the two experiments were different. The back-mutant (equivalent to $E$. coli B) growing on a lawn of $E$. coli $7 / 4$ and producing haloes was operating in an atmosphere containing indole, whereas $E$. coli B growing on a lawn of S.typhi 1808 and failing to produce a halo, was not. Exp. 3 was therefore carried out.

Exp. 3. Effect of indole on tryptophan production by Escherichia coli $B$. Salmonella typhi 1808, grown in casein medium + tryptophan, was washed once in casein medium, suspended in the same to a faint opacity and $0.5 \mathrm{ml}$. of this suspension flooded on each of four casein plates, which were then dried as usual. E. coli B was grown in casein medium, washed once and suspended in the same medium. Plates 1 and 2 carried the lawn only, plates 3 and 4 carried the lawn and drops of $\boldsymbol{E}$. coli $\mathrm{B}$ suspension. Into the lids of plates 2 and 4 filterpapers were fitted carrying 12 drops of $0.01 \mathrm{~m}$-indole. After $24 \mathrm{hr}$. of incubation there was no growth on plate 1 ; a slight film of growth on plate 2; drops without haloes on plate 3 and drops with well-marked haloes in a slight diffuse 
growth on plate 4. Haloes appeared only when there was an atmosphere containing indole. Thus $E$. coli B exuded tryptophan which could be used by $S$. typhi 1808 , but only in a detectable quantity in an atmosphere containing indole. Precisely the same result was obtained using $S$. typhi Bact. 1 as the implanted bacterium. In this case only 6 drops of indole were added to each filter-paper and the lawn was only very slightly grown. The haloes produced by $S$. typhi Bact. 1 in an atmosphere containing indole were well marked (Pl. 2, figs. 6, 7).

In both cases the increased production of tryptophan by growth in an atmosphere containing indole might well have been due to the use of this indole as a substrate of the indole $\rightarrow$ tryptophan enzyme of the bacteria. Exp. 4 was carried out to test whether typhoid bacilli growing in excess indole did in fact produce more tryptophan.

Exp. 4. The effect of indole on tryptophan synthesis by typhoid bacilli. Salmonella typhi Bact. 1 was grown in casein medium + glucose in two $100 \mathrm{ml}$. lots, in $500 \mathrm{ml}$. flasks, one containing $0.001 \mathrm{M}$-indole, i.e. 10 times more than the concentration usually used for growth. The $500 \mathrm{ml}$. flasks were incubated stagnant overnight and the contents then centrifuged. Both supernatant fluids were steam-distilled until that containing added indole was free of it by colour test (Ehrlich). They were then autoclaved and assayed for tryptophan by a similar growth method to that used in Exp. 1, using, however, on this occasion Escherichia coli $7 / 4$ as the test organism in defined medium.

The assay showed that the supernatant fluid from the culture grown without indole contained $0.4 \mu \mathrm{g}$. L-tryptophan $/ \mathrm{ml} . \pm 50 \%$, while that grown with indole contained $6.75 \mu \mathrm{g} . / \mathrm{ml} . \pm 4 \%$. It was therefore concluded that the haloes observed on plates containing indole were due to a much larger tryptophan production under these conditions. Nevertheless, it was surprising that Escherichia coli B should produce tryptophan as a nutrient available to another organism when it contained an active tryptophanase. It was, however, recalled that Fildes (1938) had shown that indole inhibited tryptophanase.

The experiments of Fildes (1938) were repeated with current materials and more refined methods (indole estimations by Spekker and standard curves). Escherichia coli $\mathrm{B}$ grown in the presence of $\mathrm{M} / 20,000$ tryptophan was washed and suspended in buffer at a concentration c. $4 \times 10^{8}$ organisms $/ \mathrm{ml}$., with $\mathrm{L}$-tryptophan equivalent to $30 \mu \mathrm{g}$. indole $/ \mathrm{ml}$. In $24 \mathrm{hr}$. $14 \mu \mathrm{g}$. indole $/ \mathrm{ml}$. had been produced, i.e. about $50 \%$ of the tryptophan had been degraded. At the same time the suspension was mixed with the same concentration of tryptophan +1 equivalent of indole $(30 \mu \mathrm{g}$. $/ \mathrm{ml}$.). In $24 \mathrm{hr}$. there was no degradation of tryptophan. Thus the tryptophanase had been entirely inhibited by 1 equivalent of indole. The presence of less than 1 equivalent of indole inhibited roughly in proportion.

The action of indole in relation to tryptophan production by typhoid bacilli and Escherichia coli is therefore twofold. As a substrate of the synthetic enzyme it causes much larger quantities of tryptophan to be produced and as an inhibitor of tryptophanase it allows tryptophan produced by $E$. coli to survive. In the syntrophic growth experiments of Winkler et al. (1952) the 
coli strain 366 produced excess indole, being blocked above indole. This excess indole caused over-production of tryptophan by coli 386 blocked below indole, and since the tryptophanase was inhibited also by the excess of indole, the tryptophan survived to act as nutrient to coli 366 .

\section{CONCLUSIONS}

It has been shown that strains of Salmonella typhi in which the indole $\rightarrow$ tryptophan enzyme is unimpaired synthesize tryptophan in excess during growth. The amount produced may be so small as to be undetectable by the methods used, but when indole in excess is added to a culture, the production of tryptophan is much greater. Experiments with Escherichia coli also show an accumulation of tryptophan in cultures containing indole. The survival of the tryptophan in this case is due to inhibition of the tryptophanase enzyme by the indole.

I am indebted to Professor D. D. Woods, Oxford, and to Professor K. C. Winkler, Utrecht, for cultures; to Professor Winkler and Dr B. A. D. Stocker, London, for literature and to Dr D. Kay for help in various ways.

\section{REFERENCES}

Findes, P. (1938). The production of indole by suspensions of Bact. coli. Biochem. J. $32,1600$.

Findes, P. (1945). The biosynthesis of tryptophan by Bact. typhosum. Brit. J. exp. Path. 26, 416.

Fuddes, P., Gladstone, G. P. \& Knight, B. C. J. G. (1933). The nitrogen and vitamin requirements of B. typhosus. Brit. J. exp. Path. 14, 189.

Fildes, P. \& Rydon, H. N. (1947). Inhibition of growth of Bact. typhosum by methyl derivatives of indole and tryptophan. Brit. J. exp. Path. 28, 211.

Gale, E. F. (1947). The assimilation of amino-acids by bacteria. J. gen. Microbiol. $1,53$.

LogIE, W. J. (1919-20), On the synthesis of tryptophane by certain bacteria and on the nature of indole formation. J. Path. Bact. 23, 224.

Tatum, E. L. \& Bonner, D. (1944). Indole and serine in the biosynthesis and breakdown of tryptophan. Proc. nat. Acad. Sci., Wash. 30, 30.

Winkler, K. C., van Doorn, A. W. J. \& Royers, A. F. M. (1952). Symbiosis of tryptophan-deficient mutants of E. coli B. Rec. Trav. chim. Pays-bas, 71, 5.

\section{EXPLANATION OF PLATES}

Plate 1

Fig. 1. Salmonella typhi $0901 \mathrm{R}$ growing on lawn of Escherichia coli $7 / 4 ; 2$ days.

Fig. 2. Same plate at 3 days; same magnification.

Fig. 3. Salmonella typhi. Bact. 1 growing on lawn of $E$. coli $7 / 4,2$ days.

Fig. 4. Same plate at 3 days; same magnification.

Fig. 5. Back-mutants of $E$. coli $7 / 4$ growing on lawn of $E$. coli $7 / 4 ; 6$ days.

Plate 2

Fig. 6. Salmonella typhi. Bact. 1 growing on lawn of $S$. typhi 1808 in air; $22 \mathrm{hr}$.

Fig. 7. S. typhi. Bact. 1 growing on lawn of S. typhi 1808 in an atmosphere containing indole; $22 \mathrm{hr}$.

(Received 24 July 1956) 
Journal of General Microbiology, Vol. 15, No. :3
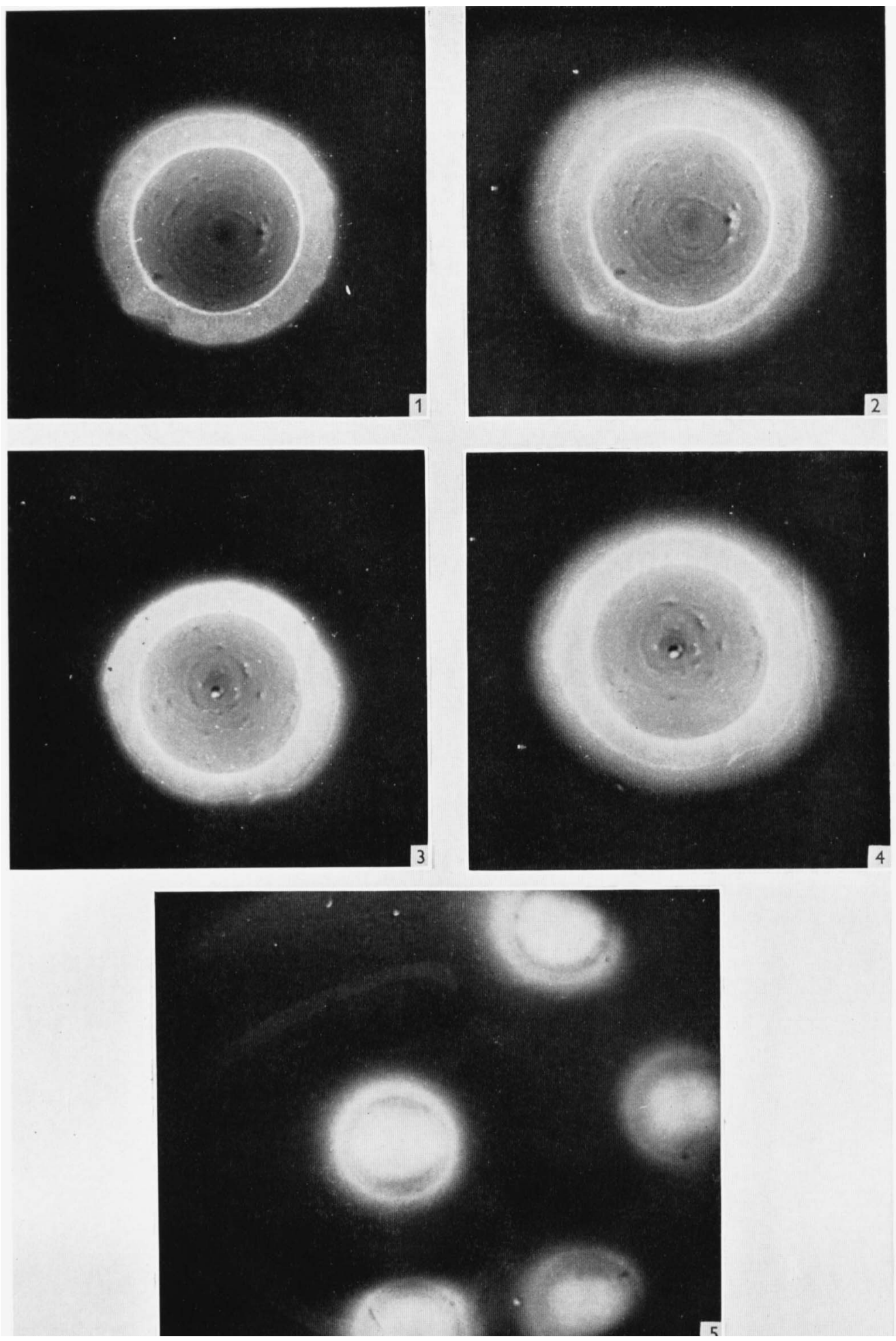

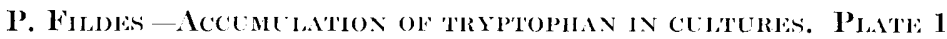


Journal of General Microbiology, Vol. 15, No. 3
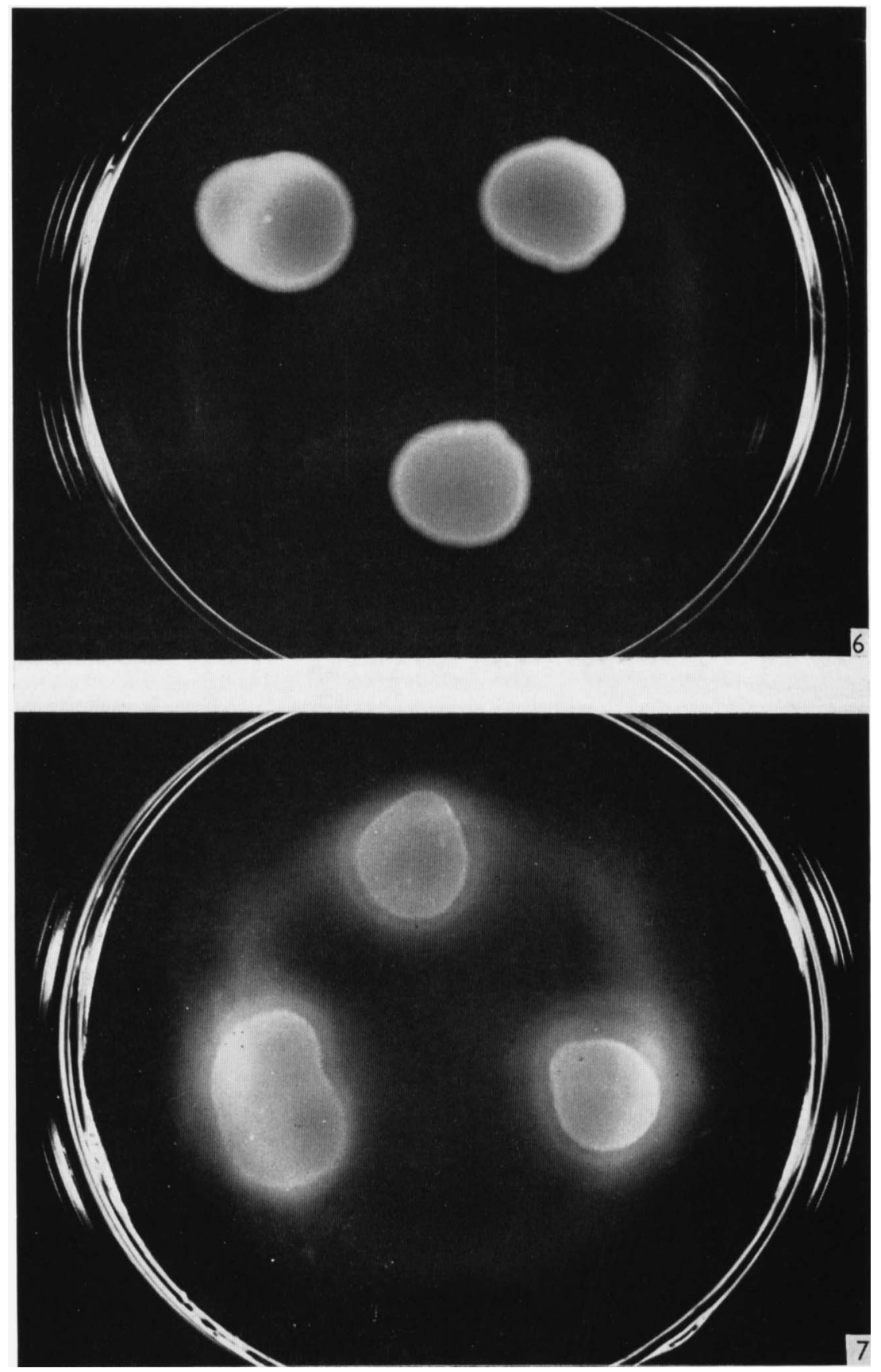

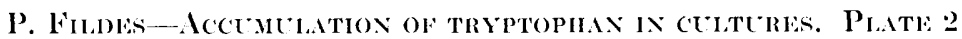

